\title{
Simulation of the spinodal phase separation dynamics of the Bi-Zn system
}

\author{
J.C.R.E. Oliveira *, M.H. Braga, R.D.M. Travasso \\ CFP and Department of Engineering Physics, R. Dr. Roberto Frias, 4200-465 Porto, Portugal \\ CEMUC and Department of Engineering Physics, R. Dr. Roberto Frias, 4200-465 Porto, Portugal \\ CFTC, Faculdade de Ciências, UL, Av. Prof. Gama Pinto, 2, 1649-003 Lisboa, Portugal
}

\section{A R T I C L E I N F O}

\section{Article history:}

Available online 1 November 2008

\section{PACS:}

61.20.Ja

61.43.Dq

65.20.De

\section{Keywords:}

Alloys

Liquid alloys and liquid metals

Diffusion and transport

Modeling and simulation

Phases and equilibria

Surfaces and interfaces

\begin{abstract}
A B S T R A C T
In the phase separation occurring at the miscibility gap (at the spinodal region) of an alloy a discrete symmetry is spontaneously broken and a domain wall network is formed. Field theory simulations are often used to study the dynamics of topological defects networks appearing in different physical contexts. In this work, we focus on the dynamics of the two immiscible liquids appearing on the phase diagram of the Bi-Zn system, one of the basic systems of lead free solders. We use phase field simulations to quantitatively simulate the dynamics of the two liquids separation in the $\mathrm{Bi}-\mathrm{Zn}$ system, at different temperatures and for different concentrations. We obtain the miscibility gap curve and the domain morphologies of the system as a function of time, temperature and component concentrations using simulations.
\end{abstract}

(c) 2008 Elsevier B.V. All rights reserved.

\section{Introduction}

Lead free solder materials are under investigation for environmental reasons. Structural and mechanical properties are of great importance in what concerns solders, in particular for the amorphous ones. In order to study the mechanical properties of amorphous solders alloys, it is crucial to study the liquid phase.

Using phase field simulations, it is possible to simulate the dynamics of immiscible liquids appearing at the miscibility gap of an alloy. These simulations may be used to determine the equilibrium compositions of the fluids for a given Gibbs energy. Even more, it is known that different parameters of the excess Gibbs energy of a certain phase may exhibit different equilibrium morphologies, in spite of the similarity of the associated equilibrium curves. Hence, these morphological studies may be an efficient method to distinguish between different Gibbs energies that give approximately the same equilibrium compositions.

The phase field simulations of solid miscibility gaps (or liquid if sufficiently rapidly quench is assumed) may also be used to determine how the mechanical properties (local stress, strain fields or Young's modulus) depend on the composition of the blend, which is determinant in what concerns solders.

\footnotetext{
* Corresponding author.

E-mail address: jespain@fe.up.pt (J.C.R.E. Oliveira).
}

In this work, we have performed phase field simulations of the two liquids separation occurring in the miscibility gap of the $\mathrm{Bi}-\mathrm{Zn}$ system for different temperatures and concentrations. We have used the Bi-Zn Gibbs energy previously calculated using the CALPHAD method and found a rich diversity of equilibrium morphologies for different points of the $\mathrm{Bi}-\mathrm{Zn}$ phase diagram. It was also possible to determine the equilibrium compositions of Liq.\#1 and Liq.\#2.

\section{Determination of the Gibbs energies using the CALPHAD method}

The CALPHAD method was used prior to this work to obtain the Gibbs energy of the liquid phase [1].

The method for the calculation of thermodynamic equilibrium is based on modeling the Gibbs energies of all coexisting phases and minimizing the total Gibbs energy of the system. The system $\mathrm{Bi}-\mathrm{Zn}$ was assessed by Malakhov [2] but some changes were introduced in the first assessment by Vizdal and co-workers [1,3].

The Gibbs energy of the regular liquid phase mixture is expressed by Vizdal et al. [1], as

$G^{\text {liquid }}=G^{\text {id }}+G^{\mathrm{E}}$,

where $G^{\mathrm{id}}=R T\left(x_{\mathrm{Bi}} \ln x_{\mathrm{Bi}}+x_{\mathrm{Zn}} \ln x_{\mathrm{Zn}}\right)$ is the ideal mixing contribution, $x$ the atomic fraction of the referred element; $G^{\mathrm{E}}=$ $x_{\mathrm{Bi}} x_{\mathrm{Zn}} \sum_{v=0}^{6}\left(x_{\mathrm{Bi}}-x_{\mathrm{Zn}}\right)^{v} \cdot{ }^{v} L_{\mathrm{Bi}, \mathrm{Zn}}$ is the contribution due to non-ideal 
interactions between the components (also known as the excess Gibbs energy of mixing) and ${ }^{v} L_{\mathrm{Bi}, \mathrm{Zn}}=a+b T$ ( $a$ and $b$ where determined using the CALPHAD method and experimental data). Other excess Gibbs energy terms can be introduced in the Gibbs energy of mixing, depending on the type of phase considered, e.g. pressure dependence, energy of the interface, energy of plastic deformation, energy of defects in the crystal lattice, etc.

\section{Numerical simulations}

The phase field method is a subject of interest since a long time ago [4-9]. Based on the Ginzburg-Landau theory of phase transitions, it shows a wide range of application - especially in materials sciences. The main feature of this method is to substitute boundary conditions at the interface by a partial differential equation for the evolution of an auxiliary field (the phase field) that takes the role of an order parameter.

In the phase separation occurring at the spinodal region of $\mathrm{Bi}-\mathrm{Zn}$ system, a mixture of Liq.\#1 and Liq.\#2 is formed. Because the mixture is incompressible we can characterize the system by one order parameter, $\phi$, which is defined as $\phi=x_{\mathrm{Zn}}-x_{\mathrm{Bi}}$. The free energy of the system can be written, phenomenologically, as a functional of $\phi$,
$F[\phi]=\int \mathrm{d} \vec{r}\left[(\nabla \phi)^{2}+G^{\text {liquid }}(\phi)\right]$,

where $G^{\text {liquid }}(\phi)$ is the energy presented in (1). The evolution of the order parameter is described by Cahn-Hilliard equation for the phase separation in solids or in viscous liquids at the diffusive regime [10]:

$\frac{\partial \phi}{\partial t}=\nabla^{2} \frac{\delta F}{\delta \phi}=-\nabla^{2}\left(\nabla^{2} \phi-\frac{\partial G^{\text {liquid }}}{\partial \phi}\right)$.

We have integrated (3) using a standard finite-difference method [11]. In Fig. 1, the miscibility gap curve of the Bi-Zn system is shown. A very good agreement, between the equilibrium curve calculated in [1] and the one obtained by the simulations was found, as expected. Here, the simulations' images for different compositions and temperatures represent the morphologies of the $\mathrm{Bi}-\mathrm{Zn}$ system at a very large dynamical range, near the equilibrium configuration. It can be seen that the size and shape of the domains changes considerably with temperature for the same concentration, and with the concentration, for the same temperature.

In Fig. 2, the evolution of the immiscible liquids with time is shown for an alloy with $x_{\mathrm{Zn}}=0.8$ at $450^{\circ} \mathrm{C}$. This is an example of the spontaneous symmetry breaking occurring inside the $\mathrm{Bi}-\mathrm{Zn}$

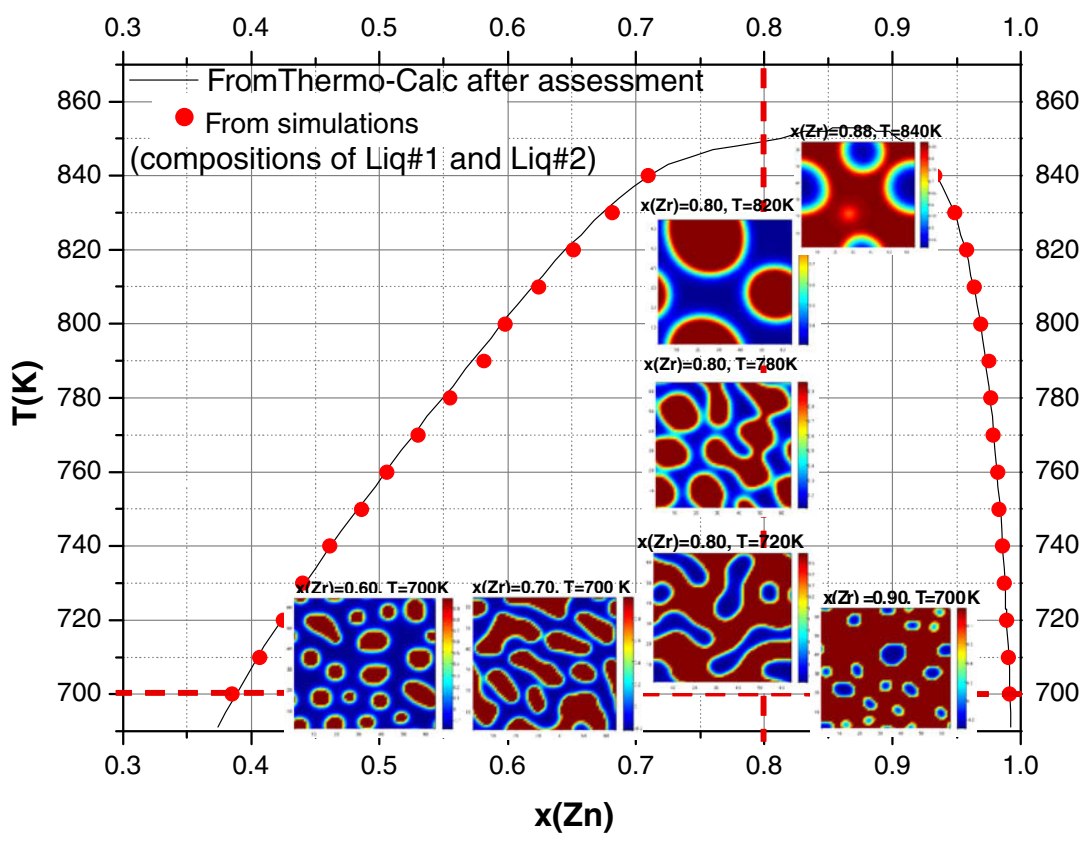

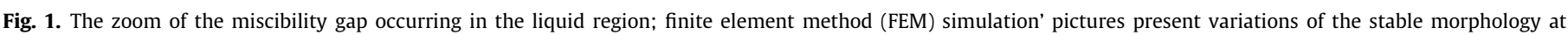

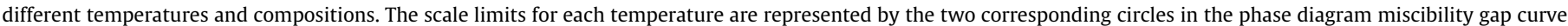
$(x($ Liquid $\# 1, \mathrm{Zn})$ and $x($ liquid\#2,Zn $)$ ).
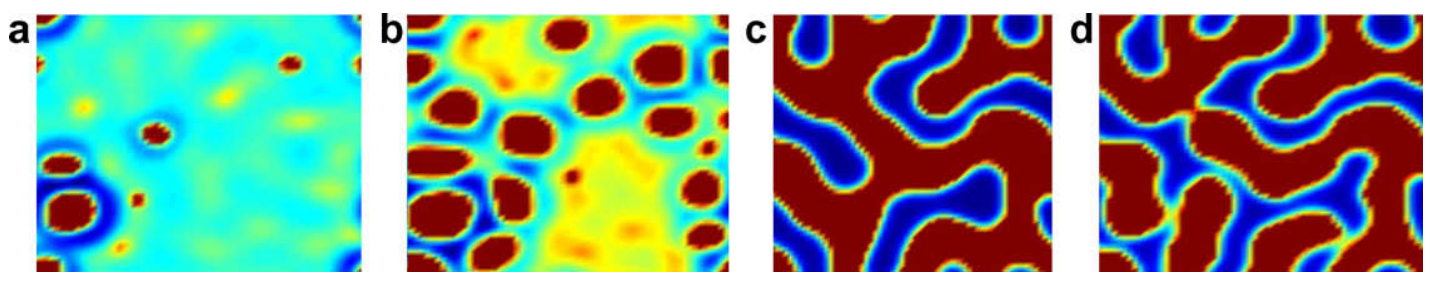

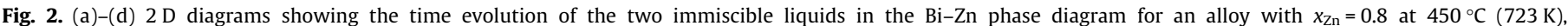

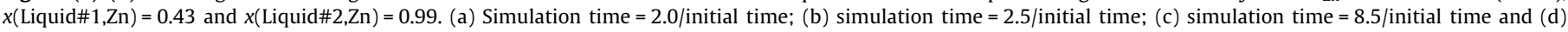
simulation time $=10 /$ initial time. 
spinodal region. It was verified that inside the spinodal region the symmetry breaks spontaneously, as expected. It was also found that with increasing temperature, the time to reach the equilibrium configurations also increases.

By the analysis of the asymptotic morphologies shown inside the miscibility gap, it can be seen that for those compositions near each side of the miscibility gap, there is a matrix of the more abundant liquid and inside this matrix, isolated round shaped domains of the other liquid phase appear. Concerning the system' compositions that are more in the middle of the spinodal region, for the same temperature as the previously referred morphologies, interconnected domains can be observed.

The analysis of the morphologies as a function of temperature, reveals that the latter interconnected domains will be transformed in spheres as the temperature rises to the upper limit of the miscibility gap. The same type of composition and temperature morphologies dependence was found in Ref. [12].

\section{Conclusions}

Using phase field simulations, the dynamics of the two immiscible liquids appearing on the phase diagram of the $\mathrm{Bi}-\mathrm{Zn}$ system was studied. It was found a very good agreement between the miscibility gap curve determined by the simulations and the one obtained by the CALPHAD method, as expected.

The different morphologies and the time it takes to reach them is a signature of the calculated excess Gibbs energy parameters for a given system.

\section{References}

[1] J. Vizdal, M.H. Braga, A. Kroupa, K.W. Richter, D. Soares, L.F. Malheiros, J. Ferreira, CALPHAD 31 (2007) 438.

[2] D.V. Malakhov, CALPHAD 24 (2000) 1.

[3] M.H. Braga, J. Vizdal, A. Kroupa, J. Ferreira, D. Soares, L.F. Malheiros, CALPHAD 31 (2007) 468

[4] J.S. Langer, H. Müller-Krumbhaar, Acta Metall. 26 (1978) 1681, 1689, 1697.

[5] A.A. Wheeler, W.J. Boettinger, G.B. Mc Fadden, Phys. Rev. A 45 (10) (1992) 7424.

[6] G. Caginalp, E. Socolovsky, SIAM J. Sci. Comput. 15 (1) (1994) 106.

[7] R. Kobayashi, Physica D 63 (1993) 410.

[8] A.A. Wheeler, B.T. Murray, R.J. Schaefer, Physica D 66 (1 \& 2) (1993) 243.

[9] J. Steinbach, F. Pezzolla, B. Nestler, M. Seeßelberg, R. Prieler, G.J. Schmitz, J.L.L. Rezende, Physica D 94 (1996) 135.

[10] A.J. Bray, Adv. Phys. 43 (1994) 357.

[11] J.C.R.E. Oliveira, C.J.A.P. Martins, P.P. Avelino, Phys. Rev. D 71 (2005) 083509.

[12] N. Nakamura, R. Takahashi, S. Sato, T. Sodesawa, S. Yoshida, Phys. Chem. Chem. Phys. 2 (2000) 4983-4990. 